\title{
Correction: Musashi RNA-binding protein 2 regulates estrogen receptor 1 function in breast cancer
}

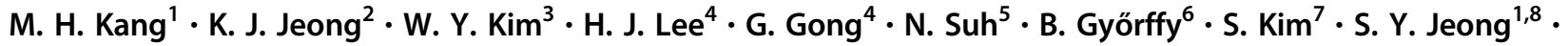 \\ G. B. Mills ${ }^{2} \cdot$ Y.-Y. Park ${ }^{1,8}$
}

Published online: 4 February 2019

(c) Springer Nature Limited 2019

\section{Correction to: Oncogene;}

https://doi.org/10.1038/onc.2016.327;

Published online 5 September 2016.

The original version of this article contained an error in Figure $2 \mathrm{e}$. In Figure $2 \mathrm{e}$, the $6^{\text {th }}$ colony image of T47D cells treated with shMSI2 was inadvertently replaced with a duplicate of $7^{\text {th }}$ colony image. However, the conclusions reported in the manuscript are not affected by figure replacement. The authors regret that these errors were made and apologize for the confusion and inconvenience. The correct version of this figure panel appears in the Author Correction associated with this Article.

These authors contributed equally: Kang MH, Jeong KJ, Kim WY

The original article can be found online at https://doi.org/10.1038/onc. 2016.327.

\section{Y.-Y. Park}

yypark@amc.seoul.kr

1 Department of Convergence Medicine, Ulsan College of Medicine, Seoul, South Korea

2 Department of Systems Biology, MD Anderson Cancer Center, Houston, TX, USA

3 Department of Surgery, Korea University, Guro Hospital, Seoul, South Korea

4 Department of Pathology, ASAN Medical Center, Seoul, South Korea

5 Department of Medicine Engineering, College of Medical Sciences, Soon Chun Hyang University, Asan-si, South Korea

6 MTA TTK Lendület Cancer Biomarker Research Group, Semmelweis University 2nd Department of Pediatrics, Budapest, Hungary

7 Anging Research Institute, Korea Research Institute of Bioscience and Biotechnology, Daejeon, South Korea

8 ASAN Institute for Life Sciences, ASAN Medical Center, Seoul, South Korea 


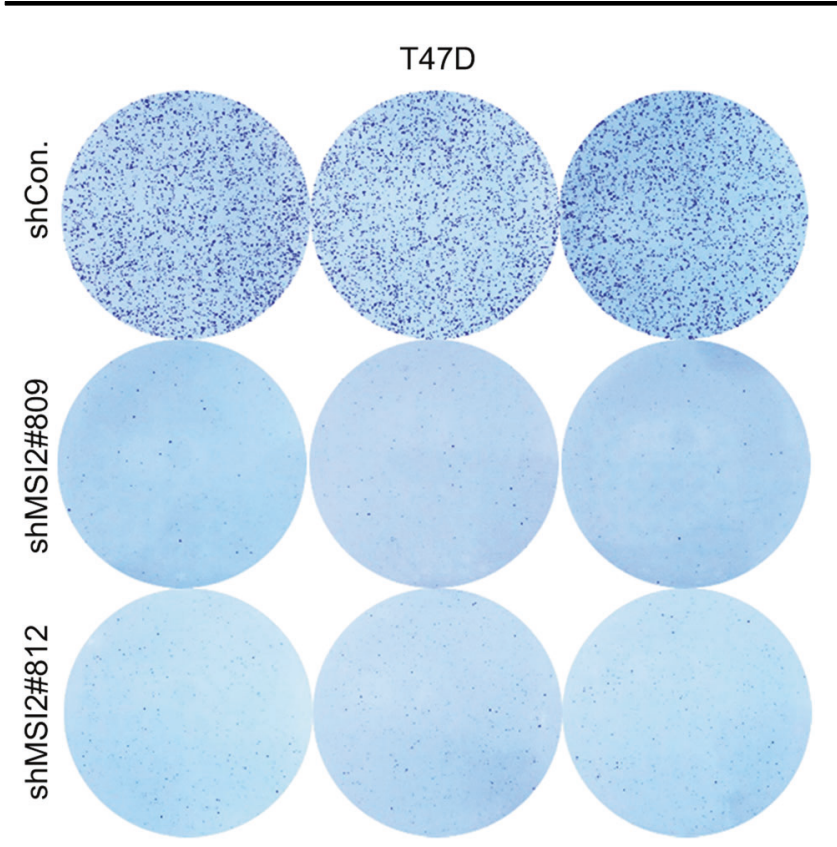

Fig. 2e 